This document is confidential and is proprietary to the American Chemical Society and its authors. Do not copy or disclose without written permission. If you have received this item in error, notify the sender and delete all copies.

\title{
Analysis of polycaprolactone microfibers as biofilm carriers for biotechnologically-relevant bacteria
}

\begin{tabular}{|r|l|}
\hline Journal: & ACS Applied Materials \& Interfaces \\
\hline Manuscript ID & am-2018-07245h.R2 \\
\hline Manuscript Type: & Article \\
\hline Date Submitted by the Author: & 29-Aug-2018 \\
\hline Complete List of Authors: & $\begin{array}{l}\text { Tamayo-Ramos, Juan Antonio; Universidad de Burgos - Campus San } \\
\text { Amaro, ICCRAM } \\
\text { Rumbo, Carlos; Universidad de Burgos, } \\
\text { Caso, Federica; Nanofaber srl } \\
\text { Rinaldi, Antonio; ENEA and University of L'Auila , UTT-MAT and MEMOCS } \\
\text { Garroni, Sebastiano; Unviersity of Burgos, ICCRAM } \\
\text { Notargiacomo, Andrea; Consiglio Nazionale delle Ricerche, } \\
\text { Romero-Santacreu, Lorena; Universidad de Burgos - Campus San Amaro } \\
\text { Cuesta-López, Santiago; ICAMCYL Foundation }\end{array}$ \\
\hline
\end{tabular}

SCHOLARONE

Manuscripts 


\title{
Analysis of polycaprolactone microfibers as biofilm carriers for biotechnologically- relevant bacteria
}

Juan Antonio Tamayo-Ramos ${ }^{1, \star}$, Carlos Rumbo ${ }^{1,3 * \$}$, Federica Caso ${ }^{4}$, Antonio Rinaldi, Sebastiano Garroni ${ }^{1}$, Andrea Notargiacomo ${ }^{6}$, Lorena Romero-Santacreu ${ }^{1,2}$, Santiago CuestaLópez $^{1,2}$

1 International Research Centre in Critical Raw Materials-ICCRAM, University of Burgos, Plaza Misael Banuelos s/n, 09001 Burgos, Spain

2 Advanced Materials, Nuclear Technology and Applied Bio/Nanotechnology. Consolidated Research Unit UIC-154. Castilla y Leon. Spain. University of Burgos. Hospital del Rey s/n, 09001 Burgos, Spain.

3 Departamento de Química, Facultad de Ciencias, University of Burgos, Plaza Misael Bañuelos s/n., 09001 Burgos, Spain

4 Nanofaber srl., Via Anguillarese 301, 00123 Rome, Italy.

5 Italian National Agency for New Technologies, Energy and Sustainable Economic Development (ENEA), Casaccia Research Centre, Via Anguillarese 301, 00123 Rome, Italy.

6 Institute for Photonics and Nanotechnology, CNR, Via Cineto Romano 42, 00156 Rome, Italy.

*Both authors contributed equally to this work.

${ }^{\$}$ Contact e-mails: ja.tamayoramos@gmail.com; crumbo@ubu.es

\begin{abstract}
Polymeric electrospun fibers are becoming popular in microbial biotechnology due to their exceptional physicochemical characteristics, biodegradability, surface-to-volume ratio, and compatibility with biological systems, which give them a great potential as microbial supports to be used in production processes or environmental applications. In this work we analyzed and compared the ability of Escherichia coli, Pseudomonas putida, Brevundimonas diminuta, and Sphingobium fuliginis to develop biofilms on different types of polycaprolactone (PCL) microfibers. These bacterial species are relevant in the production of bio-based chemicals, enzymes and proteins for therapeutic use, and bioremediation. The obtained results demonstrated that all selected species were able to attach efficiently to the PCL microfibers. Also, the ability of pure cultures of $S$. fuliginis (former Flavobacterium sp. ATCC 27551, a very relevant strain in the bioremediation of organophosphorus compounds) to form dense biofilms was observed for the first time, opening the possibility of new applications for this microorganism. This material showed to have a high microbial loading capacity, regardless of the mesh density and fiber diameter. A comparative analysis between PCL and polylactic acid (PLA) electrospun microfibers indicated that both surfaces have a similar bacterial loading capacity, but the former material showed higher resistance to microbial degradation than PLA.
\end{abstract}

\section{Keywords}


Electrospun polycaprolactone, microfibers, biofilm, bacterial attachment, biotechnology

\section{Introduction}

The application of microbial technologies in the production of high value compounds (probiotics, chemicals, enzymes, etc.), and the development of new transformation processes offering biobased solutions in multiple fields (e.g. agricultural, environmental, food, medical, etc.) have grown during the last decades and are currently experiencing a golden age. The identification and development of new microbial strains with enhanced biotransformation capacities, the need to offer bio-based solutions as an alternative to traditional processes that come with a high environmental cost, and the development of new technologies for the culture of microorganisms has fueled the interest of public and private institutions in this area. In this regard, biotransformation processes employing microorganisms involve a variety of technological approaches, that range from large scale cultivations in bioreactors (e.g. the production of a compound of interest), to their direct application in situ (e.g. bioremediation applications). In all cases, a crucial aspect is the selection of the optimal microbial culture approach and the cell (specific) surface availability to ensure the highest yield during the biotransformation process.

Most bacterial species can alternate between unicellular (planktonic) and multicellular (biofilm) states ${ }^{1}$, and both life phases can be exploited in the use of these organisms in the production of compounds of interest or in bioremediation applications. In this regard, microorganisms can be cultured through submerged (SmF), solid-state (SSF) and biofilm (BF) fermentations ${ }^{2}$. SmFs involve the growth of the organism of choice in liquid culture suspensions, while SSF and BF are surface adhesion fermentations which require the use of a support, where the microbial species grow attached to the surface. While SmF and SSF technologies have been studied in depth, BF have received less attention ${ }^{2}$, but different studies have highlighted the potential of this approach as a good alternative for SmF or SSF in bioproduction processes using bacteria and fungi ${ }^{2-5}$.

The use of solid supports for bacterial growth is also a hot topic in the bioremediation field, both for in situ and ex situ methodologies ${ }^{6}$. The inherent durability of biofilms has been studied in the treatment of different recalcitrant compounds, like halogenated compounds, hydrocarbons, pharmaceuticals residues, as well as heavy metals and toxic minerals. In biofilms, bioremediation is facilitated by enhanced gene transfer among biofilm organisms and by the increased bioavailability of pollutants for degradation, as a result of bacterial chemotaxis ${ }^{7}$.

A broad range of materials are susceptible to be used as a support for microbial growth, and they can be classified into inorganic and organic compounds. Inorganic materials used as biofilm support in bioreactors are stainless steel or ceramics, which can feature a more complex composition. The use of the latter type of materials seem favorable as they can be customized in terms of charge and pore size to comply with possible special needs of the target organism ${ }^{8}$. This is also possible in case of organic materials, which in addition can be processed in an easier way than the inorganic ones, are more flexible for modifications in terms of specific 
process needs, and are available at moderate $\operatorname{cost}^{8}$. In this regard, electrospun organic fibers have become an alternative as microorganism carriers in water bioremediation studies ${ }^{9-13}$. The attention received by this type of synthetic materials has been mainly due to their analogy with natural nanofibrous protein networks (morphology, mechanics and surface chemistry), and their high biocompatibility with human cells ${ }^{14}$, which makes them suitable to be used in human healthcare applications. These properties make them ideal surfaces for the attachment of microorganisms as well, and some research works have already studied the interaction between nanofibers and bacteria, focusing in the biomedical field ${ }^{15-17}$, and more recently in biotechnological applications ${ }^{14}$. The possibility of bacterial encapsulation during the electrospinning process has become a popular approach as well, for agriculture, food technology and bioremediation applications ${ }^{11,18-20}$.

Since the surface chemistry has been found to have a significant effect on bacterial adhesion and proliferation ${ }^{16}$, the selection of the right material depending on the desired application is a relevant matter. Electrospun fibers have been generated for several applications (wound dressings, tissue engineering, filtering processes, etc.), and from a number of synthetic and bio polymers such as nylon 6, polysulfone (PSU), polystyrene (PS), polyethylene oxide (PEO), polyacrylonitrile (PAN), polylactide (PLA), polycaprolactone (PCL), polyurethane (PU), etc. In this work, we have studied for the first time the suitability of PCL microfibers as bacterial BF support. We selected different bacterial species used in several biotechnological applications such as bioremediation, and the production of industrial enzymes and organic acids. Our aim was to study the interaction of PCL microfibers with both well-known and less studied biofilm producers, to assess their potential as BF carrying matrix in different biotech applications.

\section{Material and methods}

\section{Materials for bacteria biofilm formation}

PCL microfibers were synthesized by the company Nanofaber SRL, using the linear thermoplastic PCL diol polymer CAPA® 6800 (80,000 MW) (Prestor), chloroform (99.2\% purity, stabilized with $0.6 \%$ ethanol; VWR) and dimethylformamide (DMF) (100\% purity; VWR). Along a commercial grade called "NBARE, two variations called "Pro3" and "Pro4", were purposely provided for this investigation. These treatments were obtained from $12 \% \mathrm{PCL}$ solutions of either DMF/chloroform 2:8 (NBARE and Pro3) or pure chloroform (Pro4). The NBARE meshes are made by a mix of two kinds of microfiber with different size, while Pro3 and Pro4 meshes present a more uniform microfiber size distribution. The NBARE and its variations were benchmarked to prove robustness of bacterial conductivity towards microstructural variations. The electrospinning parameters used for the production of each PCL microfiber type are described in Table 1. The electrospun material was deposited on a flat aluminium collector.

\begin{tabular}{|l|l|l|l|l|l|l|l|l|} 
fiber & flow & applied & Moving & X axis & Y axis & spinning & depositi & microfiber \\
\hline
\end{tabular}




\begin{tabular}{|c|c|c|c|c|c|c|c|c|}
\hline type & $\begin{array}{c}\text { rate } \\
(\mu \mathrm{L} / \mathrm{h})\end{array}$ & $\begin{array}{c}\text { voltage } \\
(\mathrm{kV})\end{array}$ & $\begin{array}{c}\text { needle } \\
\text { diameter } \\
(\mathrm{mm})\end{array}$ & $\begin{array}{c}\text { speed } \\
(\mathrm{mm} / \mathrm{s})\end{array}$ & $\begin{array}{c}\text { speed } \\
(\mathrm{mm} / \mathrm{s})\end{array}$ & $\begin{array}{c}\text { distance } \\
(\mathrm{cm})\end{array}$ & $\begin{array}{c}\text { on time } \\
(\mathrm{min})\end{array}$ & $\begin{array}{c}\text { diameter } \\
(\mu \mathrm{m})\end{array}$ \\
\hline NBARE & 6000 & 23 & 1.7 & 60 & 6 & 24 & 60 & $3.80 \pm 1.08$ \\
\hline Pro3 & 4000 & 29 & 1.7 & 60 & 6 & 24 & 60 & $2.21 \pm 0.48$ \\
\hline Pro4 & 4000 & 23 & 1.7 & 60 & 6 & 24 & 60 & $6.43 \pm 1$ \\
\hline
\end{tabular}

Table 1. Process parameters used for the electrospinning of PCL solutions and microfiber diameter in the different fiber types.

PLA fibers were purchased from the company Nanopharma a.s. The obtained electrospun PCL and PLA microfibers sheets were processed with a round punch with an inner diameter of 12 $\mathrm{mm}$ to obtain round discs of the same diameter. Round glass coverslips $(12 \mathrm{~mm})$ were purchased to Thermo Scientific.

Before use, all materials were treated with $70 \%$ ethanol during 30 minutes, and afterwards irradiated with UV light for 30 minutes.

\section{Raman analysis}

The Raman spectra were acquired by means of a Alpha300R Witec employing a He-Ne laser of $532 \mathrm{~nm}$ and $20 \mathrm{~mW}$ on the sample. The spectra were recorded with $10 \mathrm{~s}$ integration time for each single spectrum and in a spectral range from ca. 3750 to $200 \mathrm{~cm}^{-1}$.

\section{Contact angle and surface roughness analysis}

The contact angle analysis of PCL microfibers was performed according to the European Standard UNI EN 15802:2009. The surface roughness of NBARE PCL mats was measured using stylus profilometry. Profilometry line-scan analysis was performed using a KLA-Tencor Alpha Step 500 Profilometer equipped with a scanning stylus with 5-micron radius tip, and $60^{\circ}$ angle, working at a stylus force of $14 \mathrm{mg}$. Line scans of $2 \mathrm{~mm}$ in length were made at a speed of $50 \mu \mathrm{m} \mathrm{s}^{-1}$.

\section{Scanning electron microscopy}

The PCL samples containing microbial biofilms for scanning electron microscopy (SEM) were obtained exactly as described above. Once the PCL microfibers were separated from the culture supernatants and were washed 3 times, they were fixed in a DPBS solution containing $2 \%$ paraformaldehyde, $2 \%$ glutaraldehyde, and $3 \mathrm{mM} \mathrm{CaCl}_{2}$. The microfibers were sequentially 
dehydrated performing 10 minute incubations in the presence of ethanol solutions of the following concentrations: 50, 70, 80, 90, 95 and 100\% (v/v). Afterwards, the samples were coated with a gold layer. Finally, the bacterial biofilms were firstly examined by with an ESEM FEI-Quanta 200F model, at the Advanced Microscopy Facility from the University of Valladolid (Spain), while the biofilm thickness was determined analyzing tilted cross-sections of the different samples, using a Jeol JSM-6460LV model, at the Microscopy Facility of the University of Burgos.

The morphological properties of PCL bare meshes NBARE, Pro3 and Pro4 were examined with a SEM Leo 1530 model (ZEISS). The samples were previously coated with a gold layer as well.

\section{Bacterial strains and culture conditions}

Three of the bacterial strains were purchased from the Spanish Type Culture Collection (CECT): P. putida CECT 4064 (DSMZ 548), B. diminuta CECT 317 (ATCC 11568), and S. fuliginis CECT 4425 (ATCC 27551). Escherichia coli DH5a was purchased from Invitrogen. All strains were maintained at $30^{\circ} \mathrm{C}$ in LB broth or agar.

\section{Crystal Violet assay}

PCL samples containing bacterial biofilms, obtained as described above, were transferred into clean wells and stained with $0.5 \%(\mathrm{w} / \mathrm{v})$ crystal violet solution (Sigma). The PCL membranes were incubated for $30 \mathrm{~min}$ at room temperature. Afterwards, the membranes were transferred to $50 \mathrm{~mL}$ tubes, and several washing steps with DPBS $(20 \mathrm{~mL})$ were performed until the washing solution was colorless (4 to 6 washing steps). Then the washed samples were transferred back to clean 24 well plates.

\section{Determination of viable bacteria adhered to the different test materials}

To study biofilm formation, static liquid micro-cultures, using $500 \mu \mathrm{L}$ of inoculated $\mathrm{LB}$, were performed on 24 well plates containing the test materials. The cultures were inoculated through a 1:100 dilution, using a pre-inoculum grown from each of the selected bacterial strains. Each pre-inoculum was obtained through the inoculation of LB $(5 \mathrm{~mL})$ with a single colony from freshly grown agar plates, followed by an overnight incubation. The micro-cultures were incubated at $30^{\circ} \mathrm{C}$ for 48 hours under static conditions. Once the culture time was finished, the materials were separated from the supernatants, rinsed three times with Dulbecco's phosphatebuffered saline (DPBS) to remove any unbound bacteria, and transferred into sterile conical tubes containing $5 \mathrm{~mL}$ of DPBS. Subsequently, to release the attached bacteria from the material the tubes were vortexed at full speed for 1 minute, placed in an ultrasonic bath, and sonicated for 15 minutes at low power. Finally, an additional 1 minute vortex step was performed, and the bacterial suspensions were serially diluted with DPBS. To determine the number of viable bacteria adhered to the different test materials, a colony forming unit count (CFU) determination was done by plating the serially diluted suspensions on LB agar plates. 
The percentage of bacteria adhered to the material and the Log CFUs $/ \mathrm{cm}^{2}$ was calculated. Three independent biological replicates were included in each assay. The data are presented as means $\pm \mathrm{SD}$.

\section{Statistical analysis}

Statistical analyses were performed using the software Prism 6.0 (GraphPad Prism, GraphPad Software, Inc.). The one-way analysis of variance (ANOVA) was used for multiple comparisons, followed by Tukey's HSD post hoc test. Differences between the attachment in PCL meshes and other materials were stablished using a Student's $t$-test. Differences were considered significant at $P \leq 0.05$.

\section{Results}

\section{Characterization of NBARE PCL microfibers}

Commercial synthetic PCL microfibers (named NBARE), provided by the company NANOFABER, were selected to study their potential as BF carrying matrix. To better understand microfiber-bacteria interactions, the surface characteristics of the electrospun material were analyzed. Firstly, the Raman spectrum of the NBARE mesh was obtained (Figure S1), and four prominent bands at $1721 \mathrm{~cm}^{-1}, 1303 \mathrm{~cm}^{-1}, 1441 \mathrm{~cm}^{-1}$ and $1107 \mathrm{~cm}^{-1}$ could be assigned to the $\mathrm{vCO}, \omega \mathrm{CH} 2, \delta \mathrm{CH} 2$ and skeletal stretching, respectively. The specific assignment of peaks are in agreement with the data reported in the literature, confirming the composition of the PCL meshes ${ }^{21}$.

The microfibers wettability was measured through a contact angle test. The water contact angle of the NBARE mats was $120.8^{\circ} \pm 5^{\circ}$, a similar result to that previously described ${ }^{22}$, showing the hydrophobic nature of the studied microfibers. The apolar behavior was confirmed by the full permeability displayed when droplets of seed oil were used to perform the test. In this case, the fluid was fully absorbed by the mesh (Figure S2).

To assess the topography of the PCL microfibers, surface roughness quantification can be done using profilometry ${ }^{23}$. Using a stylus profilometer, representative line-scan measurements were obtained (Figure S3) and values of maximum height excursion $(44.4 \mu \mathrm{m})$, average roughness $\left(R_{\mathrm{a}}, 10.5 \mu \mathrm{m}\right)$, and RMS roughness $\left(R_{\mathrm{q}}, 7.8 \mu \mathrm{m}\right)$ were calculated.

\section{Determination of biofilm formation in PCL microfibers by different bacterial strains of biotechnological interest}

The suitability of the NBARE microfibers as bacterial carriers was analyzed by studying their interaction with four different species: the laboratory strain Escherichia coli DH5 $\alpha$ (reference strain), and the biotechnology relevant strains Pseudomonas putida CECT 4064 (DSMZ 548), Brevundimonas diminiuta CECT 317 (ATCC 11568), and Sphingobium fuliginis CECT 4425 (ATCC 27551). To provide insights into the ability of these strains to develop a biofilm on PCL 
microfibers, they were grown in static liquid cultures, during 48 hours at $30^{\circ} \mathrm{C}$, in contact with the electrospun material. For this, a microculture protocol that consisted on submerging PCL microfiber discs in $500 \mu \mathrm{L}$ of the appropriate culture broth, using 24 well plates, was followed. Once the culture time ended, the fibers were washed three times with DPBS, and subsequently stained with crystal violet to visualize cell attachment. As it can be observed in Figure 1, the PCL microfibers that were submerged in sterile LB were weakly stained by crystal violet, while those submerged in liquid cultures containing each of the selected bacterial species showed to be clearly colored.

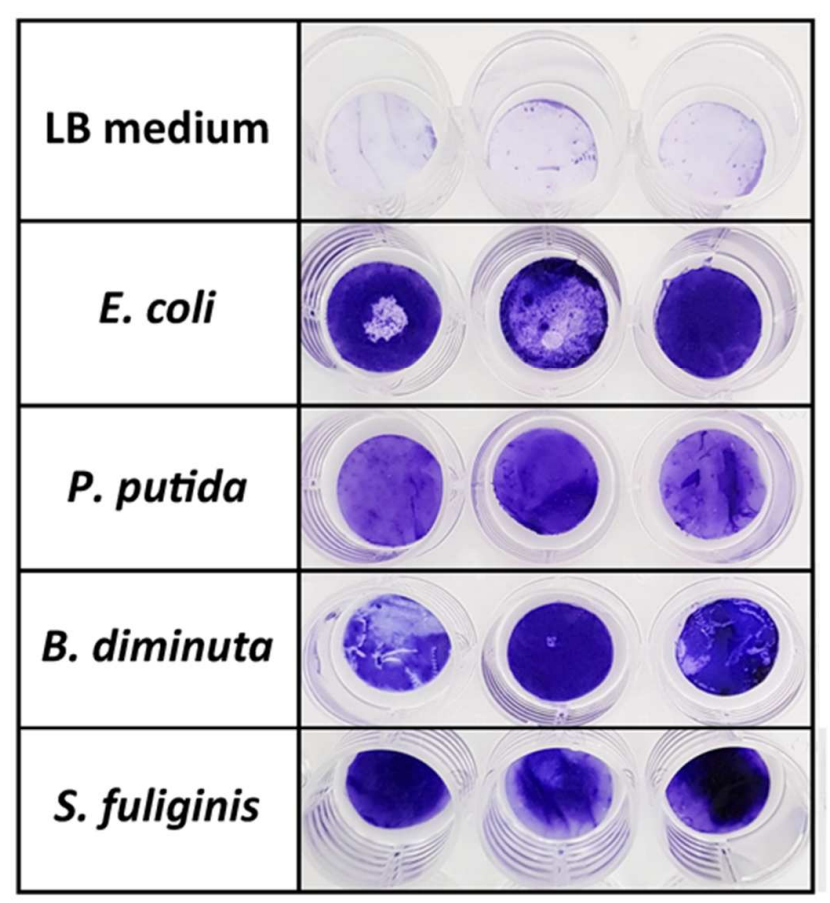

Figure 1. Visualization of microbial biofilm formation on PCL microfibers through crystal violet staining.

To confirm this result, and to visualize the biofilm formation by each of the bacterial species under study, a SEM analysis was performed too. Through the analysis of the obtained SEM micrographs the biofilm formation on the PCL fibers could be confirmed in all cases (Figure 2). The type of biofilm varied between different species. Regarding $B$. diminuta, a high density of cells was attached to the microfibers, forming tiny patches along the microfiber surface. E. coli showed a similar attachment pattern, although patches of bigger size could be observed as well in some areas of the PCL material. P. putida showed to form wide and homogeneous patches over the PCL surface, while $S$. fuliginis formed as well a dense and homogeneous biofilm. The thickness of the different microbial films could be determined by analyzing the microfibers cross- 
section, also by SEM analysis. I all cases, the films appeared to be formed by a bacterial monolayer, being the observed thickness not higher than $1 \mu \mathrm{m}$.

E. coli

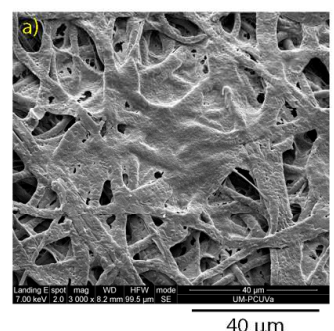

$40 \mu \mathrm{m}$

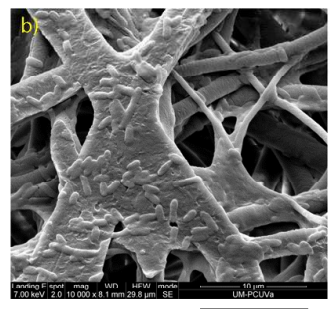

$10 \mu \mathrm{m}$
P. putida
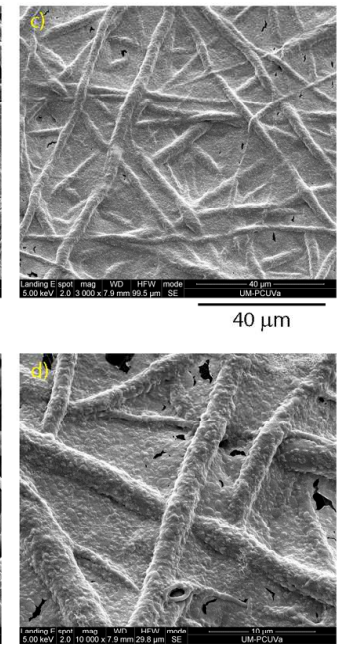

$10 \mu \mathrm{m}$
B. diminuta

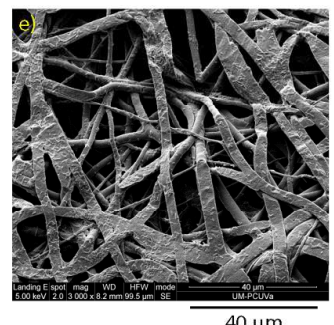

$40 \mu \mathrm{m}$

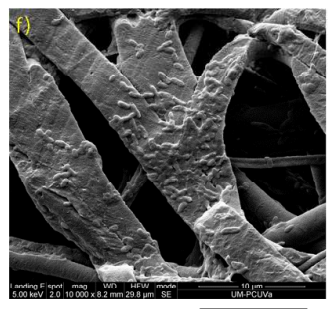

$10 \mu \mathrm{m}$
S. fuliginis
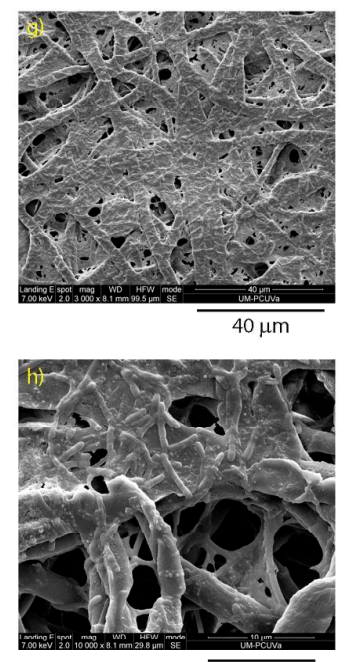

$10 \mu \mathrm{m}$

Figure 2. SEM imaging of biofilm formation by E. coli (a, b), P. putida (c, d), B. diminuta (e, f), and $S$. fuliginis $(\mathrm{g}, \mathrm{h})$ on the surface of PCL microfibers. Images on the upper row were obtained at a magnification of $3000 x$, while images on the lower row were obtained at a magnification of 10000x.

To determine the number of viable bacteria attached to the NBARE PCL fibers, the selected species were also grown in static liquid cultures in the presence of PCL discs during 48 hours at $30^{\circ} \mathrm{C}$. Afterwards, adhered bacteria were detached from the membranes by vortexing and sonication, as described in the Materials and Methods section. As it can be observed in the Figure 3 , the presence of viable bacteria attached to the PCL fibers was high in all cases, ranging from 6.4 to $8 \log \mathrm{CFUs} / \mathrm{cm}^{2}$. In average, B. diminuta showed the highest CFU counts, followed by $E$. coli, $S$ fuliginis and $P$. putida. In some cases, the differences observed were significant. $B$. diminuta showed to have significantly higher viable attached cells to $P C L$ than $P$. putida and $S$. fuliginis $(P \leq 0.01)$, while the log CFUs $/ \mathrm{cm}^{2}$ determined for $E$. coli and $P$. putida were significantly different too $(P \leq 0.05)$. 
Figure 3. Ability of different bacterial strains to attach to PCL fibers. Data represent three independent replicates. Differences were stablished using a One-way ANOVA, and considered significant at $P \leq 0.05$. ${ }^{*} P \leq 0.05,{ }^{* *} P \leq 0.01$. The error bars represent the standard deviation of values from three different biological replicates.

\section{Comparison of biofilm formation on PCL fibers with different structure}

To determine if changes on the electrospun PCL surface topography could have an impact in the bacterial attachment and subsequent biofilm formation, electrospun mats with different microfiber diameter were selected and subjected to biofilm quantification analyses. The selected microfibers, named Pro3 and Pro4, were designed with an average fiber diameter of $2.21 \mu \mathrm{m}$ and $6.43 \mu \mathrm{m}$ respectively ${ }^{24}$. Besides being significantly thinner, the Pro3 microfibers seemed to be more packed than those present in the Pro4 electrospun material, although both surfaces showed to have almost the same nominal porosity (around $80 \%$ ). The water contact angle of Pro3 and Pro4 was found to be very similar too, and comparable to that obtained for NBARE (Table S1).

The four bacterial species were grown in the presence of both PCL microfiber types, and the attachment to Pro3 and Pro4 surface was subsequently quantified (Log CFUs/ $\mathrm{cm}^{2}$ ). As displayed in Figure 4, all four species could form highly viable biofilms on both material types, with bacterial counts ranging from 6.8 to $8.4 \mathrm{log} \mathrm{CFUs} / \mathrm{cm}^{2}$ in case of Pro3, and 6.4 to $8.6 \mathrm{log}$ $\mathrm{CFUs} / \mathrm{cm}^{2}$ in case of Pro4. However, no significant differences in the capacity to form viable biofilms on both surfaces were observed for any of the selected bacterial strains. Interestingly, each of the selected species in this study showed comparable CFUs counts when grown on 
Pro3, Pro4 and NBARE (see previous experiment), confirming that electrospun PCL microfibers form a good surface for bacteria biofilm formation. Again, B. diminuta and E. coli showed the highest bacterial counts, indicating that the PCL microfiber surface is particularly suitable for the proliferation of the studied strains.

a)

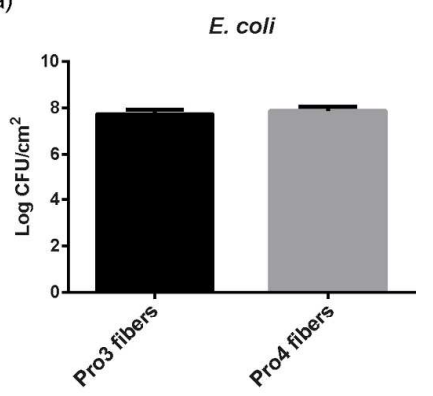

c)

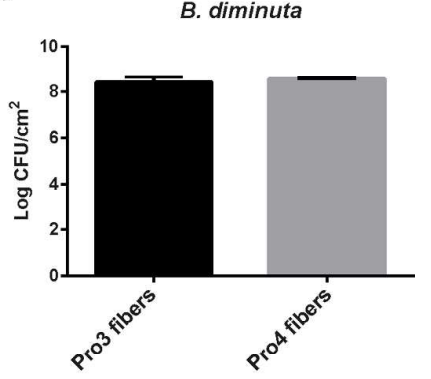

b)

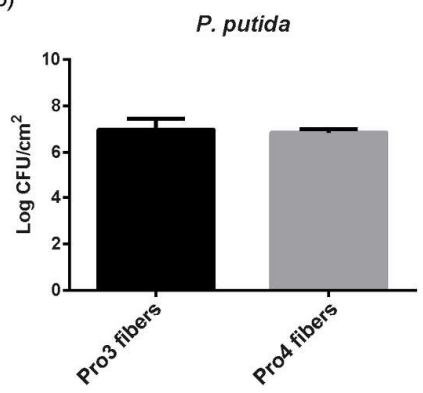

d)

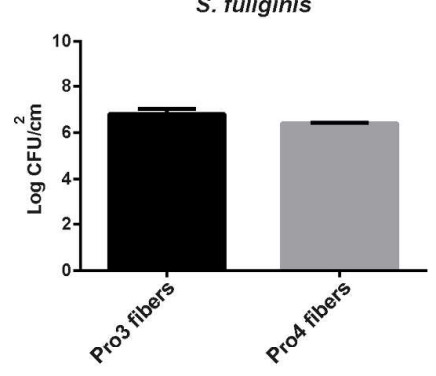

Figure 4. Log CFU $/ \mathrm{cm}^{2}$ values for E. coli (a), P. putida (b), B. diminuta (c) and S. fuliginis (d), present as a biofilm on the Pro3 (black) or the Pro4 (grey) PLC fibers. The error bars represent the standard deviation of values from three different biological replicates.

\section{Comparative analysis of biofilm formation on different materials}

The attachment ability to the NBARE PCL microfibers shown by the four selected strains was compared with their capacity to form viable biofilms on alternative surfaces. First, $B$. diminuta, $E$. coli, $P$. putida and $S$. fuliginis were grown, following the procedure described in previous sections, in the presence of NBARE PCL and glass surfaces. The attachment to electrospun $\mathrm{PCL}$ and glass was quantified (Log CFUs $/ \mathrm{cm}^{2}$ ), and different bacterial counts could be observed between both surfaces: higher microbial counts were observed on the PCL surfaces when compared to the glass coverslips, in all cases (Figure 5). Also, the selected species showed differences in the binding specificity on the two materials. Regarding B. diminuta, similar CFU 
numbers were noted for both surfaces, but still significant differences in the attachment level were observed $(P \leq 0.05)$. Differences in attachment level observed for $S$. fuliginis had the same significance level $(P \leq 0.05)$, while $P$. putida $(P \leq 0.01)$ and $E$. coli $(P \leq 0.0001)$ showed clearer differences in terms of biofilm formation between the two materials.

a)

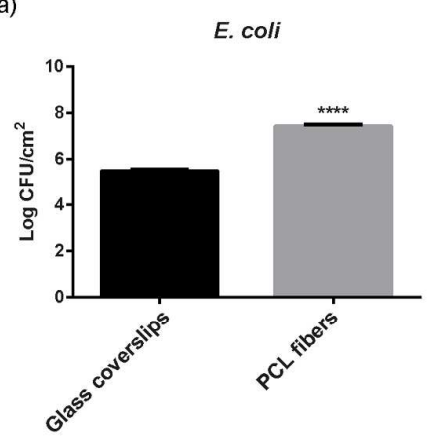

c)

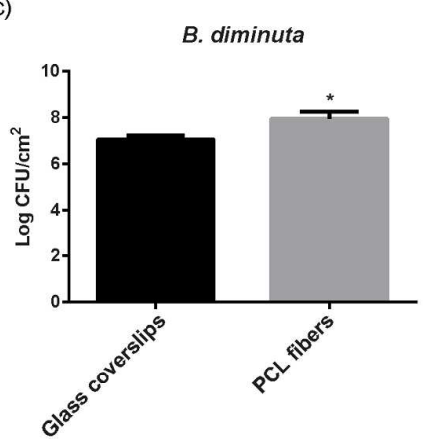

b)

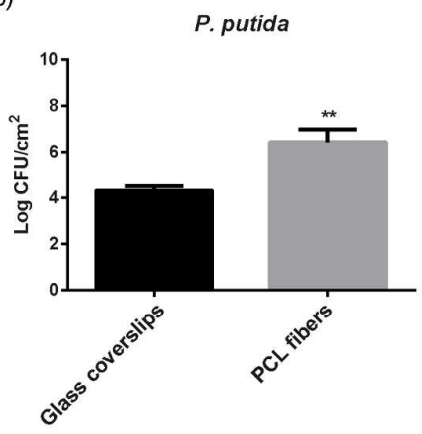

d)

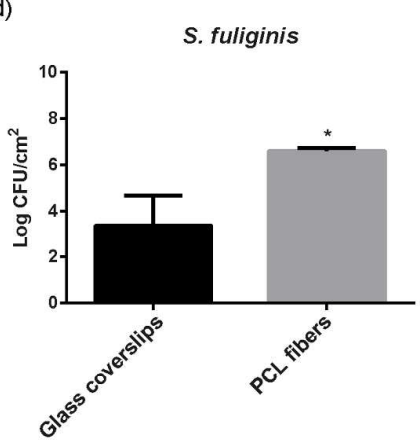

Figure 5. Viable E. coli (a), P. putida (b), B. diminuta (c) and S. fuliginis (d) counts attached (Log CFUs/cm2) to glass coverslips (black) and PCL electrospun discs (grey). Data represent three independent replicates. Differences were stablished using a Student's t-test, and considered significant at $\mathrm{P} \leq 0.05$. ${ }^{*} \mathrm{P} \leq 0.05,{ }^{* *} \mathrm{P} \leq 0.01$, ${ }^{* * * *} \mathrm{P} \leq 0.0001$. The error bars represent the standard deviation of values from three different biological replicates.

The biofilm formation ability of the selected microorganisms was compared as well by testing their ability to colonize electrospun surfaces with different chemical composition. This was done by growing all four strains in the presence of NBARE PCL and polylactic acid (PLA) microfibers. The bacterial attachment to the different microfibers was quantified and, as displayed in Figure 6 , no significant differences between the attachment levels to the distinct polymeric surfaces were noted. 
a)

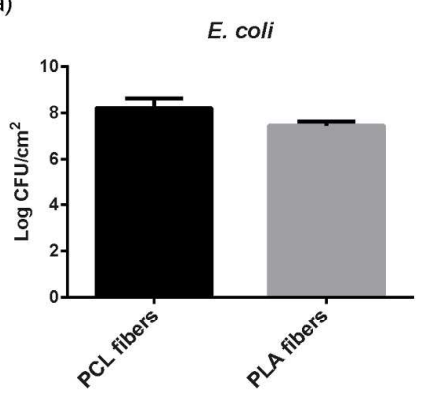

c)

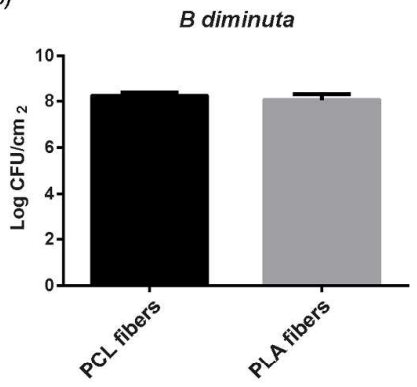

b)

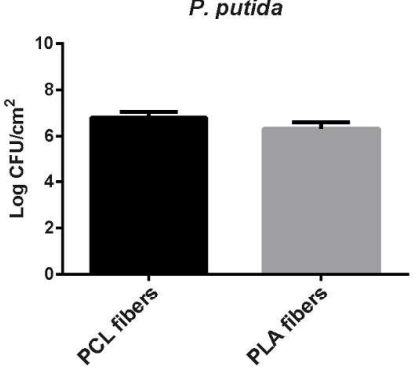

d)

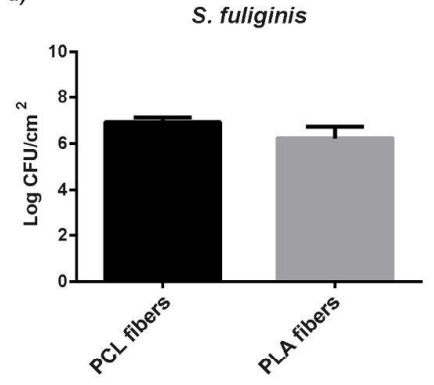

Figure 6. Log CFUs values for E. coli (a), P. putida (b), B. diminuta (c) and S. fuliginis (d), present as a biofilm on the PCL (black) or the PLA (grey) fibers. The error bars represent the standard deviation of values from three different biological replicates.

However, after bacterial detachment for CFUs quantification, clear integrity differences could be appreciated between both materials. The PCL microfibers kept their integrity in a similar state to that shown prior to the biofilm formation, while the PLA microfibers were, in most of the cases, disintegrated as displayed in Figure 7. To discard that the bacterial detachment protocol (see Material and Methods section for details) could provoke the structural deterioration of the PLA electrospun material, sterile membranes were incubated in clean LB medium and subsequently processed as usually done for CFUs quantification. The membranes keep their integrity, showing that the observed degradation phenomenon was related to the presence of the microorganisms. 
Figure 7. Integrity of PCL and PLA microfibers after bacterial biofilm detachment for CFUs quantification.

\section{Discussion}

Their exceptional physicochemical characteristics, surface-to-volume ratio, and compatibility with biological systems give electrospun fibers a great potential in biotechnological applications. To date, only few studies have explored the use of polymeric fibers as microbial cell carriers, but generally no proper controls have been used or quantitative data of cell attachment has been provided to properly estimate the cell attachment level achieved. Also, these studies have usually focused on a single material-microbial strain interaction for a specific application. In this work, we assessed the suitability of commercial synthetic PCL nanofibers as bacterial carriers by studying their interaction with four different species: the biotechnology relevant strains $P$. putida CECT 4064 (DSMZ 548), B. diminiuta CECT 317 (ATCC 11568), and S. fuliginis CECT 4425 (ATCC 27551), and the laboratory strain E. coli DH5 $\alpha$. All together, they cover a wide range of biotechnological applications, which could benefit from the use of electrospun fibers as microbial support. E. coli $\mathrm{DH} 5 \alpha$ was developed for laboratory work, and in the present study it was chosen as reference strain, as done earlier in previous studies ${ }^{25,26}$. P. putida DSMZ 548 has a high capability to degrade rather recalcitrant and inhibiting xenobiotics ${ }^{27}$, and related strains are successfully used for the production of bio-based polymers and a broad range of chemicals ${ }^{28}$. B. diminuta ATCC 11568 is a relevant coenzyme Q10 producer ${ }^{29}$, a valuable molecule for pharmaceutical and cosmetic applications ${ }^{30}$, and related strains are used as well in the production of other fine chemicals, enzymes and in bioremediation ${ }^{31-33}$. S. fuliginis ATCC 27551, previously known as Flavobacterium sp. ATCC 27551 , and related strains, are model organisms on the restoration of contaminated environments. S. fuliginis ATCC 27551 has the ability to degrade a broad spectrum of organophosphorus pesticides and insecticides, which are of great environmental concern due to their presence on contaminated soils, sediments and 
groundwater $^{33}$. For this reason, the immobilization of this strain for bioremediation purposes on magnetic nanoparticles, through covalent binding and ionic adsorption, has been studied ${ }^{34}$. The ability of E. coli, $P$. putida and $B$. diminuta strains to develop biofilms has been described before, but knowledge on $S$. fuliginis biofilm formation capacity in any surface type is very scarce. The presence of this organism in microbial communities forming biofilms ${ }^{35}$ and colonizing the roots of the aquatic plant Phragmites australis has been confirmed ${ }^{36,37}$. However, there are no previous studies reporting $S$. fuliginis biofilms developed by pure cultures on specific surfaces, being a desirable characteristic that will increment the number of its potential biotechnological applications. Therefore, the ability of the ATCC 27551 strain to form biofilms was evaluated and compared to that of E. coli, P. putida and B. diminuta, better known species for their surface colonization capacity ${ }^{31,38,39}$. Qualitative analyses using crystal violet staining and SEM visualization indicated that all bacterial species can easily colonize the NBARE PCL electrospun microfibers. The obtained results also showed that the crystal violet assay is a good method for the visualization of bacterial biofilms formed on this material. The SEM analysis allowed the visualization of the cell attachment pattern and biofilm microstructure of the selected bacterial species on PCL microfibers, including S. fuliginis. Both biofilm visualization techniques were useful to confirm the ability of the different species to attach to the studied surface, but none of these approaches gave information on the viability of the biofilms. In similar studies, where bacterial cell attachment to electrospun PS, PSU, cellulose acetate, and poly (d,Ilactide)/poly (ethylene oxide) (PDLLA/PEO) nanofibrous webs was investigated 9,12,17,40, different methods to determine the relative amount of cells alive within formed biofilms were used, as the LIVE/DEAD fluorescence assay or CFUs determination. Here, to provide quantitative data that helps to understand and compare the ability of the selected strains to develop viable biofilms on PCL microfibers, a determination of the CFUs attached to the microfibers after 48 hours of incubation, at $30^{\circ} \mathrm{C}$, was performed. The four selected strains showed a good ability to form biofilms on the NBARE PCL surface. In general, the number of bacteria recovered from the PCL microfiber discs was comparable to those obtained for good biofilm formers in previous studies, using reference surfaces, like glass, PS or steel ${ }^{41-45}$. However, the availability of studies quantifying viable bacteria in biofilms formed on electrospun fibers is currently limited, and the provided data is either qualitative ${ }^{17,40}$, or not directly comparable with the results reported here, as the reported quantitative data is expressed in different units ${ }^{12,13}$. Nevertheless, the reported results in these studies, where the biofilm formation of different bacterial species was analyzed on polymeric fibrous materials different than PCL, suggest that they could be good bacteria biofilm carriers as well.

Bacterial attachment and subsequent biofilm formation can be impacted by surface topography 46. In a recent study by Abrigo and collaborators ${ }^{17}$, the interaction of $E$. coli, Pseudomonas aeruginosa, and Staphylococcus aureus with PS nanofibers with different fiber diameters was investigated. The ability of the different strains to proliferate within the fibrous networks was affected by fiber diameter, and the highest proliferation rates occurred when fiber diameter was close to the bacterial size. However, in the present study no significant differences in biofilm 
formation were observed in PCL microfibers with different structure: NBARE (mix of two fiber types with average diameters of $3.80 \mu \mathrm{m}$ and $0.80 \mu \mathrm{m}$ ), Pro3 (average diameter of $2.21 \mu \mathrm{m}$ ) and Pro4 (average diameter of $6.43 \mu \mathrm{m}$ ) showed to have a similar bacterial load independently of the studied specie. The fact that both studies show contrasting results is not surprising, due to relevant differences in their experimental design: Abrigo and collaborators performed shaken cultures $(120 \mathrm{rpm})$ for 1 hour, while we performed static cultures for 48 hours. Therefore, the results obtained by the two studies, using distinct incubation periods, provide complementary information that helps to understand the colonization dynamics of microorganisms in surfaces with a similar structure. The results presented here are in concordance with a previous study, performed by our research group, that determined the colonization ability of different pathogenic bacterial strains on electrospun PCL fibers using long incubation times too ${ }^{24}$. Both studies show that, regardless of the above-mentioned microstructural differences displayed by NBARE, Pro3 and Pro4 (which can be visualized in the Figure S4), no variation on biofilm formation ability by different bacterial strains was observed. In this regard, it is interesting to remark that although the three electrospun PCL samples have different structure, all of them showed to have comparable wettability (Table S1), a factor that can influence as well the adhesion properties of bacteria on biomaterials, according to recent studies ${ }^{47,48}$.

Once stablished the good potential of electrospun PCL membranes as biofilm carrier surfaces for various bacterial species of biotechnological interest, we aimed to compare the performance of this type of biocompatible polymer with that of a reference surface (glass), and a similar product with a different chemical composition (PLA). Circular borosilicate glass coverslips, with the same diameter as the PCL microfiber discs, were used as reference material for the comparative analysis of biofilm formation. This material was selected as it is of standard use when studying the capacity of bacterial species to form biofilms ${ }^{44,45,49,50}$. The CFUs determined for glass and PCL surfaces indicated that the bacterial biofilms present in the electrospun material retain greater viability or are present in higher abundance. Also, the attachment degree in function of the microbial strain used was more variable on the glass surface $\left(10^{3}-10^{7} \mathrm{cfu} / \mathrm{cm}^{2}\right)$ than on the PCL surface $\left(10^{6}-10^{8} \mathrm{cfu} / \mathrm{cm}^{2}\right)$. The potential of polymeric materials as good bacterial carriers for industrial applications has been described before. This is the case of PLA surfaces, which have been previously tested as biofilm carriers in bioremediation studies ${ }^{51-53}$. This material has not been tested yet as carrier of biotechnologically relevant bacteria in the form of electrospun fibers. Therefore, to find out whether electrospun biodegradable microfibers with distinct composition could have different bacterial adhesion levels into their surface, the biofilm formation capacity of the selected species on NBARE PCL microfibers and on commercial PLA microfibers (for details about the providers see Materials and Methods) was compared. Interestingly, no significant differences in biofilm formation were observed between the NBARE PCL microfibers and the PLA microfibers. In this sense, it is remarkable that both fiber types differed not only in their chemical composition but also in their surface topography, being PLA (average diameter lower than $1 \mu \mathrm{m}$ ) significantly thinner than the NBARE PCL microfibers (mix of two fiber types with average diameter of $3.80 \mu \mathrm{m}$ and $0.80 \mu \mathrm{m}$ ). This result 
confirms that the biofilm formation of the selected strains in late colonization stages is not easily affected by the electrospun microfibers diameter.

Regarding the endurance of the materials after the bacteria biofilm formation, relevant differences were observed in the integrity of PCL and PLA microfibers. Our results showed that the structure of PLA was seriously damaged by the four bacterial strains, while this was not the case for PCL. Both polymers are biodegradable aliphatic polyesters, and certain fungal and bacterial species have shown an ability to degrade them ${ }^{54}$. The differences observed in degradation susceptibility by the selected microorganisms could have an influence on the possible applications of this type of materials when used as solid supports for biofilm formation. In case of the PCL fibers, the apparent robustness of this material shows its potential applicability in biofilm bioreactors, as a support for immobilized microorganisms.

\section{Conclusion}

This study reports the ability of several bacterial species of biotechnological interest to attach and develop biofilms in electrospun PCL microfibers. This material showed to have a high microbial loading capacity, regardless the mesh density, the fiber diameter, or the selected bacterial specie. In addition, the selected microbial strains showed to have a similar ability to form biofilms in the surface of electrospun materials with distinct composition. Remarkably, $S$. fuliginis, for which no previous reports on biofilm formation were found, showed a good ability to form high density biofilms on PCL and PLA electrospun surfaces. Finally, all three PCL microfiber types used in this study showed to have a high resistance towards microbial degradation, particularly when compared to the PLA polymeric material. The described characteristics make electrospun PCL microfibers a promising material to be used as solid supports for biotransformation processes using microbial strains.

\section{Associated content}

\section{Supplementary Information}

NBARE PCL microfibers Raman spectra (Figure S1); NBARE PCL microfibers contact angle analysis results (Figure S2); NBARE PCL microfibers profilometry line scan (Figure S3); NBARE, Pro3 and Pro4 PCL microfibers contact angle analysis data (Table S1); comparative microfiber SEM imaging of NBARE, Pro3 and Pro4 PCL microfibers (Figure S4).

\section{Conflict of interest}


The authors A. Rinaldi and F. Caso are affiliated with the company NANOFABER srl (www.nanofaber.com).

\section{Acknowledgements}

The Project has received funding from the European Union's H2020 research and innovation programme under the Marie Skłodowska-Curie grant agreement $N^{\circ} 691095$. The contracts of JATR and CRL were supported by the grants $N^{\circ}$ BU079U16 and $N^{\circ}$ BU092U16, that were cofinanced by Junta de Castilla y León and the European Social Fund.

\section{References}

(1) Berlanga, M.; Guerrero, R. Living Together in Biofilms: The Microbial Cell Factory and Its Biotechnological Implications. Microb. Cell Fact. 2016, 15 (1), 165.

(2) Gamarra, N. N.; Villena, G. K.; Gutiérrez-Correa, M. Cellulase Production by Aspergillus Niger in Biofilm, Solid-State, and Submerged Fermentations. Appl. Microbiol. Biotechnol. 2010, 87 (2), 545-551.

(3) Zohora, U. S.; Rahman, M. S.; Ano, T. Biofilm Formation and Lipopeptide Antibiotic Iturin A Production in Different Peptone Media. J. Environ. Sci. 2009, 21, S24-S27.

(4) Rahman, M. S.; Ano, T.; Shoda, M. Biofilm Fermentation of Iturin A by a Recombinant Strain of Bacillus Subtilis 168. J. Biotechnol. 2007, 127 (3), 503-507.

(5) Ercan, D.; Demirci, A. Current and Future Trends for Biofilm Reactors for Fermentation Processes. Crit. Rev. Biotechnol. 2015, 35 (1), 1-14.

(6) Edwards, S. J.; Kjellerup, B. V. Applications of Biofilms in Bioremediation and Biotransformation of Persistent Organic Pollutants, Pharmaceuticals/personal Care Products, and Heavy Metals. Appl. Microbiol. Biotechnol. 2013, 97 (23), 9909-9921.

(7) Singh, R.; Paul, D.; Jain, R. K. Biofilms: Implications in Bioremediation. Trends Microbiol. 2006, 14 (9), 389-397.

(8) Muffler, K.; Lakatos, M.; Schlegel, C.; Strieth, D.; Kuhne, S.; Ulber, R. Application of Biofilm Bioreactors in White Biotechnology. In Advances in biochemical engineering/biotechnology; 2014; Vol. 146, pp 123-161.

(9) San Keskin, N. O.; Celebioglu, A.; Sarioglu, O. F.; Ozkan, A. D.; Uyar, T.; Tekinay, T.; Wendorff, J. Removal of a Reactive Dye and Hexavalent Chromium by a Reusable Bacteria Attached Electrospun Nanofibrous Web. RSC Adv. 2015, 5 (106), 86867- 
86874.

(10) Sarioglu, O. F.; Yasa, O.; Celebioglu, A.; Uyar, T.; Tekinay, T. Efficient Ammonium Removal from Aquatic Environments by Acinetobacter Calcoaceticus STB1 Immobilized on an Electrospun Cellulose Acetate Nanofibrous Web. Green Chem. 2013, 15 (9), 2566.

(11) Sarioglu, O. F.; Keskin, N. O. S.; Celebioglu, A.; Tekinay, T.; Uyar, T. Bacteria Encapsulated Electrospun Nanofibrous Webs for Remediation of Methylene Blue Dye in Water. Colloids Surfaces B Biointerfaces 2017, 152, 245-251.

(12) Sarioglu, O. F.; Celebioglu, A.; Tekinay, T.; Uyar, T.; Syed, M. A.; Al-Madadhah, M.; Iyera, K. S. Evaluation of Contact Time and Fiber Morphology on Bacterial Immobilization for Development of Novel Surfactant Degrading Nanofibrous Webs. RSC Adv. 2015, 5 (124), 102750-102758.

(13) San Keskin, N. O.; Celebioglu, A.; Uyar, T.; Tekinay, T. Microalgae Immobilized by Nanofibrous Web for Removal of Reactive Dyes from Wastewater. Ind. Eng. Chem. Res. 2015, 54 (21), 5802-5809.

(14) Moffa, M.; Pasanisi, D.; Scarpa, E.; Marra, A. R.; Alifano, P.; Pisignano, D. Secondary Metabolite Production from Industrially Relevant Bacteria Is Enhanced by Organic Nanofibers. Biotechnol. J. 2017.

(15) Kargar, M.; Wang, J.; Nain, A. S.; Behkam, B.; Blu, T.; Unser, M.; Arciola, C. R. Controlling Bacterial Adhesion to Surfaces Using Topographical Cues: A Study of the Interaction of Pseudomonas Aeruginosa with Nanofiber-Textured Surfaces. Colloids Surf., A 2010, 364 (40), 72-81.

(16) Abrigo, M.; Kingshott, P.; McArthur, S. L. Bacterial Response to Different Surface Chemistries Fabricated by Plasma Polymerization on Electrospun Nanofibers. Biointerphases 2015, 10 (4), 04A301.

(17) Abrigo, M.; Kingshott, P.; McArthur, S. L. Electrospun Polystyrene Fiber Diameter Influencing Bacterial Attachment, Proliferation, and Growth. ACS Appl. Mater. Interfaces 2015, 7 (14), 7644-7652.

(18) Salalha, W.; Kuhn, J.; Dror, Y.; Zussman, E. Encapsulation of Bacteria and Viruses in Electrospun Nanofibres. Nanotechnology 2006, 17 (18), 4675-4681.

(19) López-Rubio, A.; Sanchez, E.; Sanz, Y.; Lagaron, J. M. Encapsulation of Living Bifidobacteria in Ultrathin PVOH Electrospun Fibers. Biomacromolecules 2009, 10 (10), 2823-2829.

(20) De Gregorio, P. R.; Michavila, G.; Ricciardi Muller, L.; de Souza Borges, C.; Pomares, 
M. F.; Saccol de Sá, E. L.; Pereira, C.; Vincent, P. A. Beneficial Rhizobacteria Immobilized in Nanofibers for Potential Application as Soybean Seed Bioinoculants. PLoS One 2017, 12 (5), e0176930.

Qin, C.-C.; Duan, X.-P.; Wang, L.; Zhang, L.-H.; Yu, M.; Dong, R.-H.; Yan, X.; He, H.-W.; Long, Y.-Z. Melt Electrospinning of Poly(lactic Acid) and Polycaprolactone Microfibers by Using a Hand-Operated Wimshurst Generator. Nanoscale 2015, 7 (40), 16611-16615.

Lin, Y.-J.; Cai, Q.; Li, Q.-F.; Xue, L.-W.; Jin, R.-G.; Yang, X.-P. Effect of Solvent on Surface Wettability of Electrospun Polyphosphazene Nanofibers. J. Appl. Polym. Sci. 2010, 115 (6), 3393-3400.

(23) Guimarães, A.; Martins, A.; Pinho, E. D.; Faria, S.; Reis, R. L.; Neves, N. M. Solving Cell Infiltration Limitations of Electrospun Nanofiber Meshes for Tissue Engineering Applications. Nanomedicine 2010, 5 (4), 539-554.

(24) Rumbo, C.; Tamayo-Ramos, J. A.; Caso, M. F.; Rinaldi, A.; Romero-Santacreu, L.; Quesada, R.; Cuesta-López, S. Colonization of Electrospun Polycaprolactone Fibers by Relevant Pathogenic Bacterial Strains. ACS Appl. Mater. Interfaces 2018, acsami.7b19440.

(25) Hou, S.; Burton, E. A.; Simon, K. A.; Blodgett, D.; Luk, Y.-Y.; Ren, D. Inhibition of Escherichia Coli Biofilm Formation by Self-Assembled Monolayers of Functional Alkanethiols on Gold. Appl. Environ. Microbiol. 2007, 73 (13), 4300-4307.

(26) Wood, T. K.; González Barrios, A. F.; Herzberg, M.; Lee, J. Motility Influences Biofilm Architecture in Escherichia Coli. Appl. Microbiol. Biotechnol. 2006, 72 (2), 361-367. Monteiro, Á. A. M. .; Boaventura, R. A. .; Rodrigues, A. E. Phenol Biodegradation by Pseudomonas Putida DSM 548 in a Batch Reactor. Biochem. Eng. J. 2000, 6 (1), 45-49.

(28) Poblete-Castro, I.; Becker, J.; Dohnt, K.; dos Santos, V. M.; Wittmann, C. Industrial Biotechnology of Pseudomonas Putida and Related Species. Appl. Microbiol. Biotechnol. 2012, 93 (6), 2279-2290.

(29) Processes for Producing Coenzyme Q10. 2007.

(30) Cluis, C. P.; Pinel, D.; Martin, V. J. The Production of Coenzyme Q10 in Microorganisms. In Sub-cellular biochemistry; 2012; Vol. 64, pp 303-326.

(31) Li, X. Z.; Hauer, B.; Rosche, B. Catalytic Biofilms on Structured Packing for the Production of Glycolic Acid. J. Microbiol. Biotechnol. 2013, 23 (2), 195-204.

(32) Warda, E. A.; Abeer, A. A. E. A.; Eman, R. H.; Mahmoud, A. S.; Ahmed, I. E.-D.; Warda, E. A.; Abeer, A. A. E. A.; Eman, R. H.; Mahmoud, A. S.; Ahmed, I. E.-D. Applications of 
Plackett-Burman and Central Composite Design for the Optimization of Novel Brevundimonas Diminuta KT277492 Chitinase Production, Investigation of Its Antifungal Activity. Brazilian Arch. Biol. Technol. 2016, 59 (0).

(33) Singh, B. K.; Walker, A. Microbial Degradation of Organophosphorus Compounds. FEMS Microbiol. Rev. 2006, 30 (3), 428-471.

(34) Robatjazi, S. M.; Shojaosadati, S. A.; Khalilzadeh, R.; Farahani, E. V.; Balochi, N. Immobilization of Magnetic Modified Flavobacterium ATCC 27551 Using Magnetic Field and Evaluation of the Enzyme Stability of Immobilized Bacteria. Bioresour. Technol. 2012, 104, 6-11.

(35) Al-Mailem, D. M.; Kansour, M. K.; Radwan, S. S. Bioremediation of Hydrocarbons Contaminating Sewage Effluent Using Man-Made Biofilms: Effects of Some Variables. Appl. Biochem. Biotechnol. 2014, 174 (5), 1736-1751.

(36) Toyama, T.; Momotani, N.; Ogata, Y.; Miyamori, Y.; Inoue, D.; Sei, K.; Mori, K.; Kikuchi, S.; Ike, M. Isolation and Characterization of 4-Tert-Butylphenol-Utilizing Sphingobium Fuliginis Strains from Phragmites Australis Rhizosphere Sediment. Appl. Environ. Microbiol. 2010, 76 (20), 6733-6740.

(37) Toyama, T.; Ojima, T.; Tanaka, Y.; Mori, K.; Morikawa, M. Sustainable Biodegradation of Phenolic Endocrine-Disrupting Chemicals by Phragmites Australis -rhizosphere Bacteria Association. Water Sci. Technol. 2013, 68 (3), 522.

(38) Pratt, L. A.; Kolter, R. Genetic Analysis of Escherichia Coli Biofilm Formation: Roles of Flagella, Motility, Chemotaxis and Type I Pili. Mol. Microbiol. 1998, 30 (2), 285-293.

(39) López-Sánchez, A.; Leal-Morales, A.; Jiménez-Díaz, L.; Platero, A. I.; Bardallo-Pérez, J.; Díaz-Romero, A.; Acemel, R. D.; Illán, J. M.; Jiménez-López, J.; Govantes, F. Biofilm Formation-Defective Mutants in Pseudomonas Putida. FEMS Microbiol. Lett. 2016, 363 (13), fnw127.

(40) Ahire, J. J.; Hattingh, M.; Neveling, D. P.; Dicks, L. M. T. Copper-Containing Anti-Biofilm Nanofiber Scaffolds as a Wound Dressing Material. PLoS One 2016, 11 (3), 1-12.

(41) Joseph, B.; Otta, S. K.; Karunasagar, I.; Karunasagar, I. Biofilm Formation by Salmonella Spp. on Food Contact Surfaces and Their Sensitivity to Sanitizers. Int. J. Food Microbiol. 2001, 64 (3), 367-372.

(42) Marques, S. C.; Rezende, J. das G. O. S.; Alves, L. A. de F.; Silva, B. C.; Alves, E.; Abreu, L. R. de; Piccoli, R. H. Formation of Biofilms by Staphylococcus Aureus on Stainless Steel and Glass Surfaces and Its Resistance to Some Selected Chemical Sanitizers. Brazilian J. Microbiol. 2007, 38 (3), 538-543. 
(43) Silagyi, K.; Kim, S.-H.; Martin Lo, Y.; Wei, C. Production of Biofilm and Quorum Sensing by Escherichia Coli 0157:H7 and Its Transfer from Contact Surfaces to Meat, Poultry, Ready-to-Eat Deli, and Produce Products. Food Microbiol. 2009, 26 (5), 514-519.

(44) Coraça-Huber, D. C.; Fille, M.; Hausdorfer, J.; Putzer, D.; Nogler, M. Efficacy of Antibacterial Bioactive Glass S53P4 against S. Aureus Biofilms Grown on Titanium Discs in Vitro. J. Orthop. Res. 2014, 32 (1), 175-177.

(45) Greene, C.; Wu, J.; Rickard, A. H.; Xi, C. Evaluation of the Ability of Acinetobacter Baumannii to Form Biofilms on Six Different Biomedical Relevant Surfaces. Lett. Appl. Microbiol. 2016, 63 (4), 233-239.

(46) Feng, G.; Cheng, Y.; Wang, S.-Y.; Borca-Tasciuc, D. A.; Worobo, R. W.; Moraru, C. I. Bacterial Attachment and Biofilm Formation on Surfaces Are Reduced by SmallDiameter Nanoscale Pores: How Small Is Small Enough? npj Biofilms Microbiomes 2015, 1 (1), 15022.

(47) Yuan, Y.; Hays, M. P.; Hardwidge, P. R.; Kim, J. Surface Characteristics Influencing Bacterial Adhesion to Polymeric Substrates. RSC Adv. 2017, 7 (23), 14254-14261.

(48) Wassmann, T.; Kreis, S.; Behr, M.; Buergers, R. The Influence of Surface Texture and Wettability on Initial Bacterial Adhesion on Titanium and Zirconium Oxide Dental Implants. Int. J. Implant Dent. 2017, 3 (1), 32.

(49) Han, H.; Wu, J.; Avery, C. W.; Mizutani, M.; Jiang, X.; Kamigaito, M.; Chen, Z.; Xi, C.; Kuroda, K. Immobilization of Amphiphilic Polycations by Catechol Functionality for Antimicrobial Coatings. Langmuir 2011, 27 (7), 4010-4019.

(50) Salas-Jara, M.; llabaca, A.; Vega, M.; García, A. Biofilm Forming Lactobacillus: New Challenges for the Development of Probiotics. Microorganisms 2016, 4 (4), 35.

(51) Lai, Z. N.; Cui, Y. De; Gao, P.; Chen, X. J. Modified PLA Carrier Material and Its Performance in Immobilization of Nitrifying Bacteria. Mater. Sci. Forum 2009, 610-613, 198-201.

(52) Fan, Z.; Hu, J.; Wang, J. Biological Nitrate Removal Using Wheat Straw and PLA as Substrate. Environ. Technol. 2012, 33 (21), 2369-2374.

(53) Walczak, M.; Swiontek Brzezinska, M.; Sionkowska, A.; Michalska, M.; Jankiewicz, U.; Deja-Sikora, E. Biofilm Formation on the Surface of Polylactide during Its Biodegradation in Different Environments. Colloids Surfaces B Biointerfaces 2015, 136, 340-345.

(54) Tokiwa, Y.; Calabia, B. P.; Ugwu, C. U.; Aiba, S. Biodegradability of Plastics. Int. J. Mol. Sci. 2009, 10 (9), 3722-3742. 
Table of contents graphic

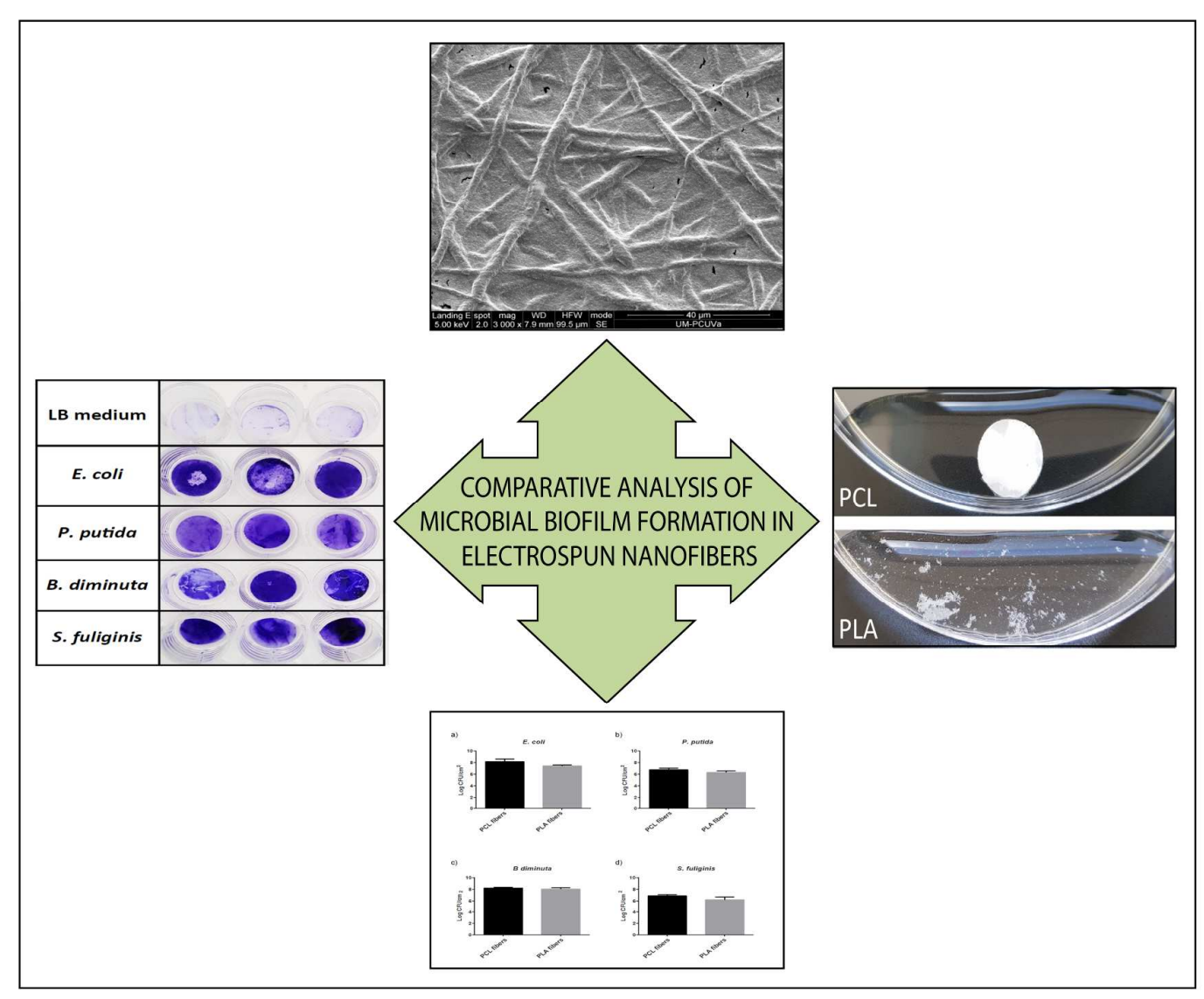

